\title{
Identité nationale et relations communautaires à travers l'œuvre poétique de Kóstas Montis
}

National Identity and Communities Relations through the Poems of

Costas Montis

\section{Alexandre Lapierre}

\section{OpenEdition}

\section{Journals}

Édition électronique

URL : https://journals.openedition.org/ceb/8854

DOI : $10.4000 /$ ceb.8854

ISSN : 2261-4184

\section{Éditeur}

INALCO

Édition imprimée

ISBN : 978-2-85831-229-0

ISSN : 0290-7402

\section{Référence électronique}

Alexandre Lapierre, «Identité nationale et relations communautaires à travers l'œuvre poétique de Kóstas Montis », Cahiers balkaniques [En ligne], 43 | 2015, mis en ligne le 24 juillet 2017, consulté le 06 juillet 2021. URL : http://journals.openedition.org/ceb/8854 ; DOI : https://doi.org/10.4000/ceb.8854

Ce document a été généré automatiquement le 6 juillet 2021.

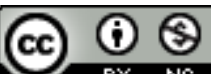

Cahiers balkaniques est mis à disposition selon les termes de la Licence Creative Commons Attribution - Pas d'Utilisation Commerciale 4.0 International. 


\title{
Identité nationale et relations communautaires à travers l'œuvre poétique de Kóstas Montis
}

\author{
National Identity and Communities Relations through the Poems of \\ Costas Montis
}

Alexandre Lapierre

\section{Introduction}

1 La poésie à Chypre est un genre populaire pratiqué depuis l'Antiquité ${ }^{1}$. Les auteurs les plus célèbres se sont souvent inspirés de la tradition orale des joutes poétiques improvisées ${ }^{2}$ ( $\left.\tau \sigma \alpha \tau \tau i ́ \sigma \mu \alpha \tau \alpha ; \varepsilon \rho \omega \tau \imath \kappa \alpha ́\right)$ par des poètes autodidactes, pour créer une production profondément ancrée dans l'actualité de l'île et dans ses préoccupations. Ils le font parfois crûment, comme on peut le constater. Nous pouvons ainsi observer dans les textes de Kóstas Montis ou d'Andreas Pastellas la ferveur nationaliste des années de lutte pour l'indépendance. Cette poésie évolue dans ses thématiques et sa symbolique, mettant en avant tantôt l'affirmation communautaire, tantôt la douleur et la colère, et, plus récemment, les espoirs de réunification et l'identité commune entre Chypriotes grecs et turcs dans la nostalgie d'un passé révolu (idéalisé peut-être?) où les deux communautés vivaient en bonne intelligence.

2 Montis est un auteur emblématique de Chypre puisque c'est l'un des Chypriotes les plus connus, correspondant de l'Académie d'Athènes, nommé pour le Prix Nobel de littérature, (il est au programme des enseignements de littérature en Grèce), mais aussi l'un des plus engagés en faveur de l'indépendance de Chypre. Il est très connu pour sa

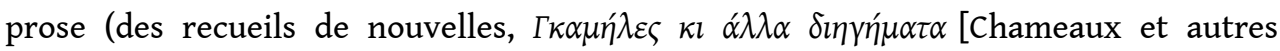

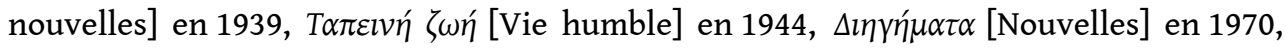

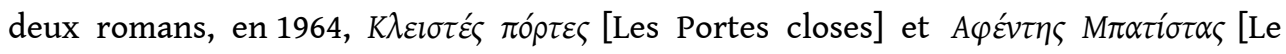


Baptiste], roman de 1984), mais également pour sa poésie riche d'une dizaine de recueils.

3 Montis, est né en 1914 à Famagouste ; son histoire personnelle est, à l'image de celle de Chypre, marquée dans une certaine mesure par la mort, le déchirement, et la déception ${ }^{3}$. Il perd sa mère à l'âge de 11 ans, quelques années plus tard deux de ses frères, et son père, à l'âge de 16 ans. Après des études primaires et secondaires dans l'île, il entreprend des études de Droit à Athènes, à une époque où l'administration britannique ne permet pas l'exercice des professions d'avocat à ceux qui ont fait leur Droit en Grèce. Ce choix n'est pas anodin, car il espère qu'avant la fin de ses études l'union avec la Grèce sera réalisée. La déception de cet espoir le marque durablement, y compris dans son art. À la même époque, il est correspondant à Athènes pour le journal chypriote Eleftheria sous le nom d'emprunt de Kóstas Alkimos. À son retour à Chypre, il trouve un emploi d'administrateur dans une compagnie minière grecque. Puis il devient enseignant à l'école de comptabilité de Kyrenia. En 1942, il s'installe à Nicosie et crée avec Achilléas Lymbourides et Phivos Moussoulides le premier théâtre professionnel de Chypre, Lyriko. Jusqu'en 1946, il publie avec la collaboration de Moussoulides le périodique The Theater. Cette même année, il intègre la Chambre de commerce chypriote dont il édite le journal. Il publie aussi le journal Elefthera Phoni jusqu'en 1947 avant d'intégrer le journal Ethnos un an plus tard. En 1950, il devient secrétaire général de la Chambre de commerce de Chypre. De 1956 à 1969, il est en charge du cahier littéraire du magazine Time of Cyprus. En 1961, il prend ses fonctions de Directeur du Tourisme, fonction qu'il occupe durant 15 ans avant de prendre sa retraite en 1976. Si Montis a commencé sa carrière par la rédaction de pièces de théâtre, son œuvre est particulièrement riche par la variété des textes et des styles. Il a composé pour le théâtre, mais il est aussi connu pour ses contes, sa poésie, pour enfants notamment, ses romans, rédigés en grec démotique, en anglais, mais aussi dans la première langue qu'il ait parlée, le dialecte chypriote. Reconnu comme une "voix littéraire de Chypre». Il a été nommé pour le prix Nobel en 1984, et reconnu correspondant de l'Académie d'Athènes en 2000.

4 Lorsqu'éclate la lutte pour l'indépendance de Chypre en 1955, Montis s'engage dans le combat de l'EOKA, il est d'ailleurs guide politique des membres de l'organisation à Nicosie. Il n'est donc pas étonnant qu'il ait choisi de rédiger sur ce sujet un roman, les Portes closes. L'œuvre poétique de Montis est très marquée par l'engagement identitaire de la communauté chypriote mais l'auteur y fait part aussi de ses doutes et de sa douleur au gré des épreuves traversées par le peuple de Chypre. Montis a en effet vécu toutes les périodes marquantes de l'histoire récente de Chypre, sa poésie en est un reflet.

5 Aussi ai-je choisi d'articuler cette intervention autour de trois thématiques qui structurent le questionnement identitaire au sein de la communauté grecque de Chypre : la ferveur nationale, le doute, et pour finir la douleur et la colère.

\section{La ferveur nationale}

6 Cette ferveur pour la libération nationale est mise en chants par de jeunes poètes, membres de l'EOKA, au nombre desquels compte Michalakis Karaolis dont certains vers

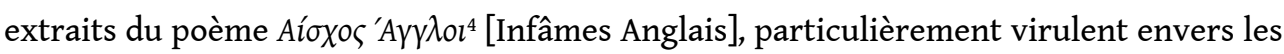
Anglais, sont repris dans un chant de l'organisation. Karaolis est l'incarnation du 
sacrifice des jeunes combattants de l'EOKA, c'est en effet le premier d'entre eux à être pendu en mai 1956 par les Anglais.

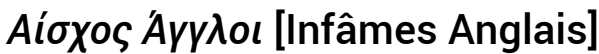

\begin{tabular}{|c|c|}
\hline 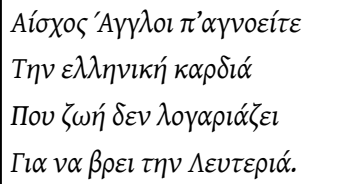 & $\begin{array}{l}\text { Infâmes Anglais qui ignorez } \\
\text { le cœur grec } \\
\text { qui ne compte pas sa vie } \\
\text { quand il s'agit de sa Liberté. }\end{array}$ \\
\hline 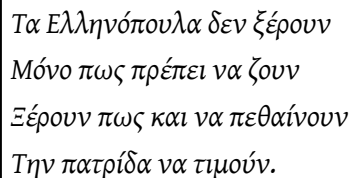 & $\begin{array}{l}\text { Les Jeunes Grecs ne savent pas } \\
\text { seulement qu'ils doivent vivre } \\
\text { ils savent aussi qu'ils doivent mourir } \\
\text { pour honorer la patrie. }\end{array}$ \\
\hline
\end{tabular}

$7 \quad$ Montis cite Karaolis par son prénom dans les Portes closes sans pour autant s'attarder sur ses faits d'armes ni sur l'importance du jeune homme - alors âgé d'une vingtaine d'années - au sein de l'organisation. Il insiste en revanche sur son sacrifice et son supplice.

\section{Premier extrait des Portes closes}

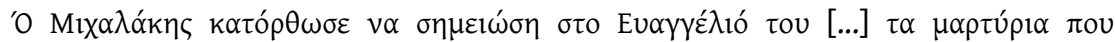

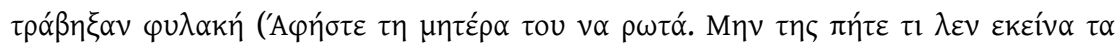

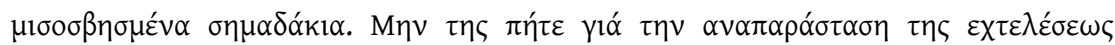

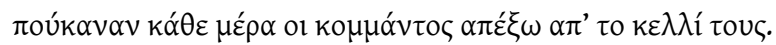

Kíx!

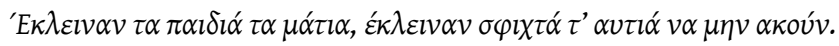

Eh you, kih !)

Michalakis a réussi à écrire des notes dans sa Bible [...] sur les tortures endurées en détention (Laissez sa mère à ses questions. Ne lui dites pas la signification de ces signes à peine lisibles. Ne lui parlez pas des simulacres d'exécutions que les commandos faisaient chaque jour en dehors de leurs cellules.

Kih!

Les enfants fermaient les yeux, ils se bouchaient les oreilles pour ne pas entendre.

Eh you, kih !) 5

Il célèbre aussi Evagóras Pallikarídis à plusieurs reprises dans ses recueils, et notamment ces quelques vers ${ }^{6}$, dans lesquels il semble regretter un manque de courage chez lui et les autres poètes, alors que le jeune Pallikarídis a délaissé la protestation poétique pour l'action et qu'il s'avance vers la potence... à moins qu'il ne condamne un geste vain dicté par l'innocence de la jeunesse.

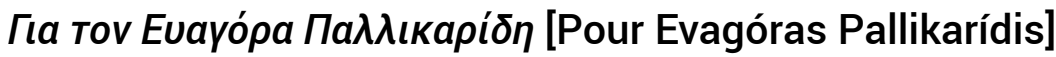

\begin{tabular}{|c|c|}
\hline 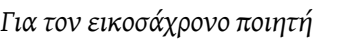 & Pour le poète de vingt ans \\
\hline Ev $\alpha \gamma o ́ \rho \alpha \Pi \alpha \lambda \lambda_{\imath \kappa \alpha \rho i ́ \delta \eta}$ & Evagóras Pallikarídis \\
\hline 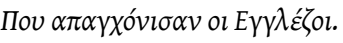 & Que les Anglais ont pendu. \\
\hline
\end{tabular}




\begin{tabular}{|c|c|}
\hline 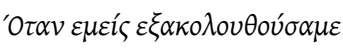 & Alors que nous continuions \\
\hline 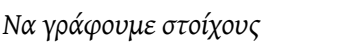 & À rédiger des vers, \\
\hline 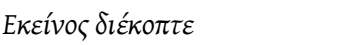 & Lui s'est interrompu \\
\hline 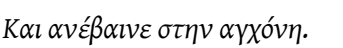 & Pour monter à la potence. \\
\hline
\end{tabular}

9 Pallikarídis est né le 28 février 1938 dans la région de Paphos, où il a effectué ses études primaires et secondaires. Il entre de manière spectaculaire dans la résistance en sabotant une cérémonie en l'honneur de la reine Élizabeth en juin 1955. Il quitte les bancs du lycée de Paphos la même année pour intégrer les rangs de l'EOKA. Le 18 décembre 1956, il est arrêté par la police britannique en compagnie de deux camarades. L'équipe transporte du ravitaillement et des armes. Pallikarídis, qui détient en plus une arme lourde, est conduit en détention pour être jugé. Malgré les protestations de la Grèce et des demandes de grâce formulées par ses camarades de lycée, il est exécuté le 13 mars 1957, à peine âgé de 19 ans. C'est le plus jeune mais aussi le dernier combattant de l'EOKA à avoir été exécuté par les autorités britanniques.

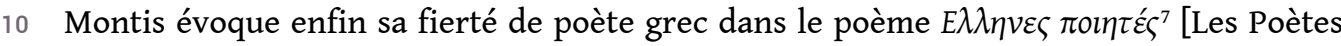

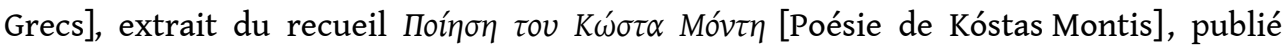
en 1962. Il y souligne aussi le sentiment de solitude et d'abandon des habitants grecs de cette île éloignée de la Mère-Patrie.

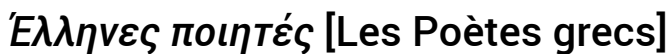

\begin{tabular}{|c|c|}
\hline 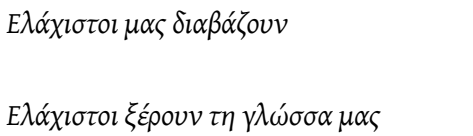 & $\begin{array}{l}\text { Très peu nombreux sont ceux qui savent notre langue } \\
\text { Très peu nombreux ceux qui nous lisent }\end{array}$ \\
\hline 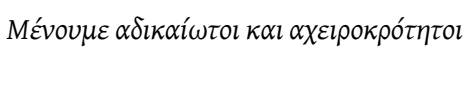 & $\begin{array}{l}\text { Nous vivons sans que l'on nous ait rendu justice, sans } \\
\text { applaudissements }\end{array}$ \\
\hline 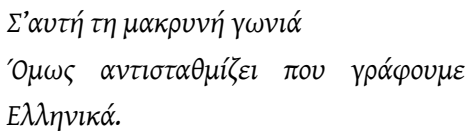 & $\begin{array}{l}\text { Dans ce lieu éloigné, } \\
\text { Mais ceci est compensé par le fait que nous écrivons en Grec. }\end{array}$ \\
\hline
\end{tabular}

\section{Le doute}

11 Le doute dans l'expression littéraire apparaît peu de temps après la liesse de l'indépendance, avec les tragiques événements de l'hiver 1963-1964. C'est un choc pour de nombreux auteurs qui entrevoient dans l'escalade des troubles un risque de destruction de la société chypriote. Certains évoquent ainsi une remise en question de leur engagement nationaliste, et émettent des doutes sur l'avenir de leur île. C'est le cas de Montis dans les Portes closes. Il y décrit certes la ferveur du peuple chypriote grec pour l'Enosis (union à la Grèce), mais évoque aussi à la fin de l'ouvrage des doutes sur l'issue d'un combat qui risque de se transformer en gâchis pour le peuple chypriote. La conclusion de l'ouvrage est assez évocatrice à ce sujet, l'histoire que nous conte Montis ne se termine pas bien puisque seule la mère reste survivante, et qu'elle n'a plus de raison d'exister puisque sa famille a disparu. 


\section{Deuxième extrait des Portes closes}

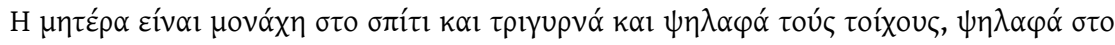

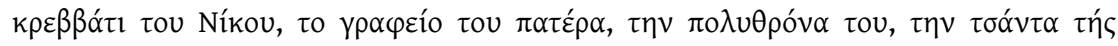

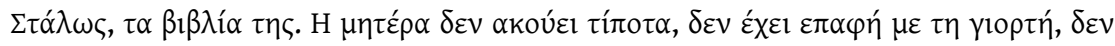

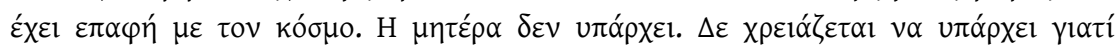

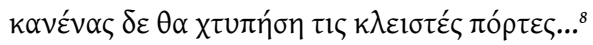

La mère est seule à la maison, elle tourne en rond et tâte les murs, elle touche le lit de Nikos, le bureau du père, son fauteuil, le sac de Stalo, ses livres. La mère n'entend rien, elle n'a pas de contact avec la fête, elle n'a pas de contact avec le monde. La mère n'existe pas. Elle n'a plus de raisons d'exister car personne ne viendra frapper aux portes closes... intercommunautaires ayant justifié l'intervention de la Force des Nations Unies à Chypre, il apporte donc une réponse non seulement à Durrell, mais aussi à l'actualité tragique de l'île. C'est par cette phrase aux intonations prémonitoires qu'il rappelle la bonne entente passée entre les deux communautés, et interpelle la communauté internationale et ses responsabilités.

\section{Troisième extrait des Portes closes}

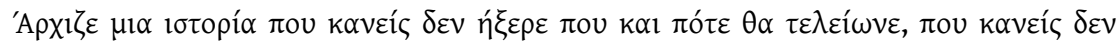

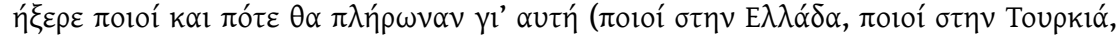

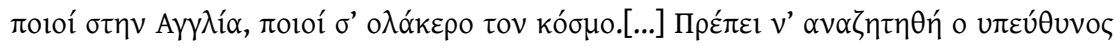

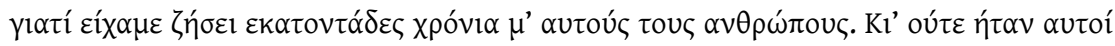

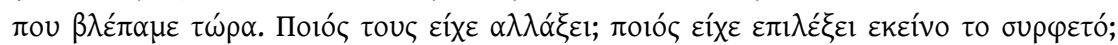
'H

Une histoire commençait dont personne ne savait où ni quand elle se terminerait, dont personne ne savait quand ni qui devrait payer pour cela (Qui en Grèce, qui en Turquie, qui en Angleterre, qui dans le Monde entier. [...] Le responsable doit être recherché car nous avions vécu plusieurs siècles avec ces gens. Mais à présent ils n'étaient plus ceux que nous avions connus. Qui les avait changés? Qui avait levé ce ramassis de gens suspects? Savait-il avec quel feu il jouait?)

Il est aussi présent dans les Lettres à la Mère. Ces lettres sont une correspondance fictive entre Montis et sa mère, dans laquelle il n'hésite pas à nous livrer son ressenti, dans des textes sombres. Cette correspondance prend la forme de trois longs poèmes publiés respectivement en 1965 (Première Lettre à la Mère), en 1972 (Deuxième Lettre à la Mère), et en 1980 (Troisième Lettre à la Mère).

\section{Deuxième Lettre à la Mère}

Mère, tout ceci est un cercle vicieux, mère, nous sommes tous un cercle vicieux, une vaste plaisanterie.

nous sommes assis à nos pupitres d'écoliers et nous écoutons les orateurs nous mentir nous sommes assis sur les bancs

et nous écoutons les orateurs dont nous savons qu'ils mentent et nous les écoutons ces orateurs 
qui savent combien nous savons qu'ils mentent...

... Et voilà la révolution

Qui ne change rien...

... En fait il n'y a pas de révolution,

Il n'y a pas de «nouveau système

... Mère tu ne reconnaîtras pas l'île.

... Mère je t'ai parlé au début d'une plaie

En fait, cette plaie c'est notre espoir, Mère

Cette plaie saignante

Cette plaie vivante.

Cela te paraît singulier, mais c'est ainsi.

Et elle est de plus en plus saignante, de plus en plus vivante...

(Euvres complètes, p. 900)

\section{Troisième Lettre à la Mère}

15 Cette troisième et dernière Lettre à la Mère publiée en 1980 est lourde de la colère et de la douleur provoquée par les événements tragiques de l'été 1974.

Je te le signifie autrement : « avec violence »

Avec violence, avec tristesse, plaie, mère...

\section{La douleur et la colère}

Dix ans après l'indépendance (1960) Chypre sombre définitivement dans la violence. Montis qui a soutenu le combat de l'EOKA (pas de l'EOKA-B il faut le préciser) et la

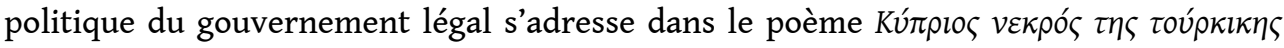
$\varepsilon i \sigma \beta o \lambda \eta \zeta^{10}{ }^{10}$ [Le mort chypriote de l'invasion turque] aux combattants morts au cours des événements. Il y souligne le caractère presque dérisoire de ce conflit au regard des grands problèmes du monde; l'honneur d'une telle mort n'aurait finalement qu'une répercussion locale et donc limitée, qui s'intéresse au sort des Chypriotes?

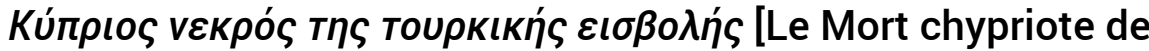 l'invasion turque]}

\begin{tabular}{|c|c|}
\hline 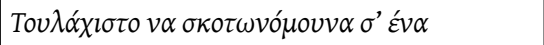 & Si au moins j'étais tué au cours \\
\hline 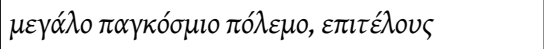 & d'une grande guerre mondiale, enfin. \\
\hline$M \alpha \sigma \kappa o \tau \omega \theta \eta \eta \kappa \alpha \sigma^{\prime} \varepsilon \dot{v} \alpha$ & Mais j'ai été tué dans une petite guerre \\
\hline$\mu \iota \kappa \rho o ́ ~ \kappa \imath^{\prime} \alpha \sigma \eta^{\prime} \mu \alpha v \tau \eta \varsigma \chi \omega ́ \rho \alpha \varsigma$, & d'un pays insignifiant, \\
\hline$\pi \circ v \delta \varepsilon v \xi_{\varepsilon} \rho \omega \alpha \nu \theta^{\prime} \alpha \nu \varepsilon \gamma \varepsilon i ́ p \eta \kappa \alpha \nu$ & dont je ne sais s'il érigera même \\
\hline 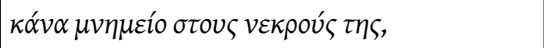 & un monument à ses morts \\
\hline 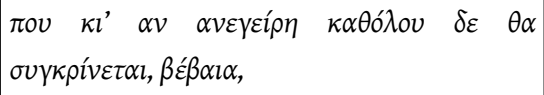 & $\begin{array}{l}\text { et s'il érige quelque-chose cela ne sera pas comparable, } \\
\text { bien entendu, }\end{array}$ \\
\hline $\begin{array}{l}\mu \varepsilon \tau^{\prime} \quad \alpha v \tau i ́ \sigma \tau o l \chi \alpha \quad \mu \nu \eta \mu \varepsilon i ́ \alpha \\
\chi \omega \rho \omega ́ \nu,\end{array}$ & avec les autres monuments des grands pays \\
\hline $\begin{array}{l}\mu \varepsilon \tau^{\prime} \alpha v \tau i ́ \sigma \tau o l \chi \alpha \quad \mu \nu \eta \mu \varepsilon i ́ \alpha \\
\pi \circ \lambda \varepsilon \dot{\mu} \mu \omega \nu\end{array}$ & avec les autres monuments des grandes guerres, \\
\hline
\end{tabular}




\begin{tabular}{|c|c|}
\hline 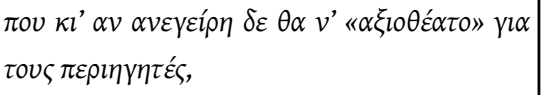 & $\begin{array}{l}\text { et s'il érige quelque-chose il ne «méritera " pas d'être } \\
\text { vu pour les voyageurs, }\end{array}$ \\
\hline 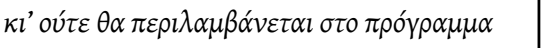 & et il ne sera même pas inclus au programme \\
\hline 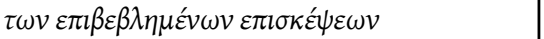 & des visites imposées \\
\hline 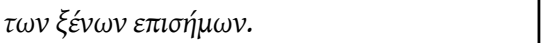 & des officiels étrangers. \\
\hline
\end{tabular}

17 Montis consacre un grand nombre de poèmes à l'invasion turque dont le titre

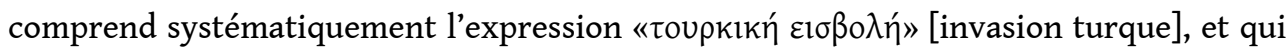
recouvrent tous les aspects du drame humain de l'invasion. C'est ainsi par exemple l'attente insoutenable des mères de portés disparus, certaines ont attendu plusieurs mois, à venir patienter au centre de Nicosie au check-point de Ledra Palace ${ }^{11}$ avant de pouvoir revoir maris ou fils déportés en Turquie, d'autres attendent toujours aujourd'hui.

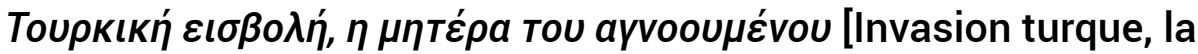 mère du porté disparu]}

\begin{tabular}{|c|c|}
\hline 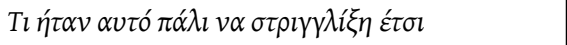 & Qu'avait-elle à nouveau à crier sa douleur ainsi \\
\hline 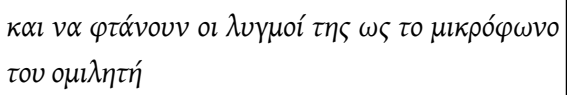 & $\begin{array}{l}\text { au point que ses sanglots parvenaient au micro de } \\
\text { l'orateur }\end{array}$ \\
\hline$\kappa \alpha l v \alpha \varepsilon \pi \varepsilon \mu \beta \alpha_{i}^{\prime} v o v v \kappa \alpha l v \alpha \sigma \nu \mu \mu \varepsilon \tau \alpha \delta i \delta \omega v \tau \alpha \alpha$ & $\begin{array}{l}\text { au point qu'ils s'ingéraient, au point qu'ils se } \\
\text { greffaient au discours }\end{array}$ \\
\hline 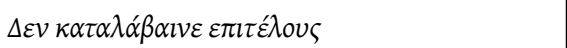 & Ne comprenait-elle pas enfin \\
\hline 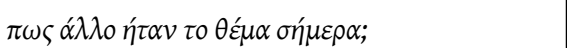 & qu'aujourd'hui le sujet était tout autre? \\
\hline
\end{tabular}

18 L'évocation poétique des lieux perdus est constante chez les auteurs de Montis à Passiardis. C'est particulièrement le cas de la chaîne de Kyrénia et du Pentadactylos. Montis consacre à cette montagne caractéristique par sa forme plusieurs poèmes, évoquant notamment l'amertume et le désespoir et une certaine forme de fatalisme

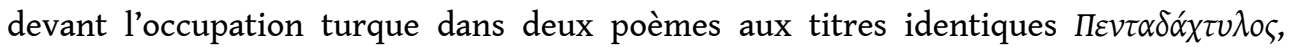

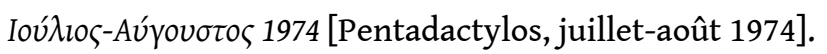

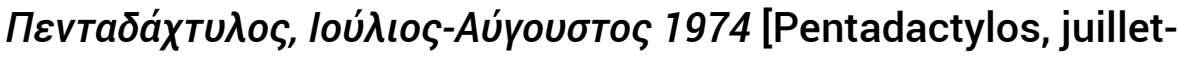 août 1974]}

\begin{tabular}{|c|c|}
\hline 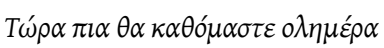 & Nous allons maintenant nous asseoir toute la journée \\
\hline$\alpha v \tau \imath \kappa \rho v ́$ o $\varepsilon^{\prime} \alpha \varsigma \sigma \tau o v \alpha \dot{\lambda} \lambda_{0}$ & L'un face à l'autre \\
\hline$v \alpha \kappa o l \tau \alpha \zeta o ́ \mu \alpha \sigma \tau \varepsilon \pi \varepsilon \rho i ́ \lambda v \pi \alpha$ & Pour nous observer tristement? \\
\hline
\end{tabular}




\begin{tabular}{|c|c|}
\hline 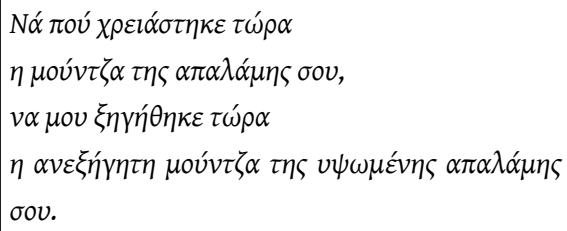 & $\begin{array}{l}\text { À quoi nous sert maintenant } \\
\text { l'injure de ta paume ouverte, } \\
\text { Que l'on m'explique maintenant } \\
\text { l'injure inexpliquée de ta paume ouverte levée vers le } \\
\text { ciel. }\end{array}$ \\
\hline 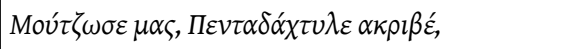 & Tu peux nous injurier, oui toi Pentadactylos, \\
\hline 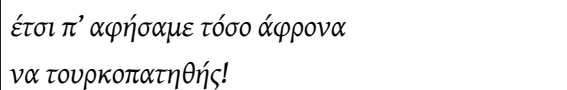 & $\begin{array}{l}\text { toi que nous avons laissé si imprudemment } \\
\text { tomber aux mains des Turcs! }\end{array}$ \\
\hline
\end{tabular}

19 L'identité et la perception communautaire se ressentent considérablement du traumatisme de 1974, elles conditionnent le positionnement de la communauté grecque vis-à-vis de la communauté turque. Les sentiments de victimisation et d'injustice entretenus dans la mémoire collective des Chypriotes grecs engendrent leur hostilité à l'égard de l'autre partie. Le souvenir des bombardements et de l'invasion, issue tragique de la rivalité inter-ethnique, encourage dans les esprits l'idée que la communauté turque et son soutien, la Turquie, constituent une menace. L'expérience des réfugiés est particulièrement marquante dans ce cas et elle traumatise les familles sur plusieurs générations. Il faut cependant souligner que c'est plus à l'encontre des colons venus de Turquie depuis 1974 que les attitudes négatives s'expriment ${ }^{12}$.

À la différence d'autres auteurs, Montis ne semble pas trop insister sur les responsabilités locales. Il dénonce cependant la multiplication des images commémoratives, des inaugurations de monuments après 1974, qui le laissent dubitatif. Dans le poème «La Statue non-dévoilée ${ }^{13}$, publié en 1974 dans son anthologie de la poésie chypriote, l'auteur s'attaque au monde des décideurs, au caractère vain de certaines politiques qui ont conduit l'île au drame. La réification du narrateur et de sa communauté en forme de statue insiste sur la manipulation dont, pense Montis, le peuple chypriote fait l'objet. Nous pouvons y voir l'évocation des nombreuses constructions de monuments commémoratifs à la mémoire des combattants de l'EOKA, mais aussi après les événements de 1974, une multiplication qui banalise tous ces monuments, finalement oubliés par les politiques qui les ont commandés, préoccupés par d'autres soucis. Nous remarquons aussi une analogie troublante avec un monument édifié au centre de Nicosie et qui apparaît dans le documentaire de Michel Cacoyannis, «Attila 1974 ». Cette statue commémorant la libération du peuple chypriote de l'esclavage devait être inaugurée, lorsqu'eut lieu le coup d'État de juillet 1974, et l'invasion turque, elle ne fut jamais inaugurée et resta couverte de draps, c'est ainsi qu'elle nous apparaît dans le documentaire de Cacoyannis, avec en commentaire Monseigneur Makarios III expliquant que cette statue ne sera dévoilée que lorsque Chypre sera libérée du joug étranger.

\section{La Statue non dévoilée}

Une statue, au bas de la rue, voici ce que nous sommes,

et nous avons attendu longtemps pour qu'ils nous inaugurent.

Un hiver et un été nous avons attendu

et maintenant deux hivers et deux étés, à attendre

Le drap qui nous recouvre est passé,

et déchiré non loin de nos yeux.

Les officiels sont très occupés nous a-t-on dit, 
nous ne sommes pas, nous a-t-on dit, une priorité

les récents troubles ont mis au jour de nombreuses statues, nous a-t-on dit.

Nous comprenons cela. Pourquoi se sont-ils tant pressés à nous installer,

Pourquoi n'y ont-ils pas pensé avant de nous disperser ainsi ?

Si on en vient à cela ils n'ont même pas besoin d'être officiels :

Si seulement il y avait quelque esprit chrétien pour tirer le drap,

si seulement quelque enfant malicieux venait jouer avec,

si seulement les gitans venaient une nuit le voler.

Oublions l'idée qu'il puisse rester

assez longtemps pour suivre petit à petit

l'horrible progrès des loques

que le vent enlève ;

Oublions l'idée qu'il puisse rester

jusqu'à ce que le temps l'ait détruit,

jusqu'à ce que les passants soient fatigués,

jusqu'à ce que leur curiosité soit fatiguée,

jusqu'à ce qu'ils ne regardent plus

jusqu'à ce que cela n'ait plus aucune importance

qui est découvert

jusqu'à ce que cela n'ait plus aucune importance

ce que nous représentons.

Montis est en revanche particulièrement virulent envers la politique internationale, et notamment l'action des États-Unis. Il s'adresse particulièrement au représentant de leur politique étrangère au début des années 1970, le secrétaire d'État Henry Kissinger. Montis souligne sa responsabilité dans l'affaire de Chypre à plusieurs reprises. Dans le

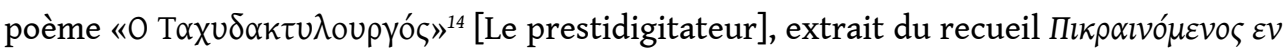
$\varepsilon \alpha v \tau \omega ́$ [Amer en moi même], publié en 1975, l'auteur s'attaque à la malice du diplomate américain sur un ton assez désabusé.

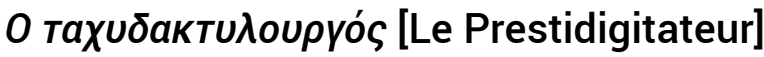

\begin{tabular}{|c|c|}
\hline$\Sigma \tau o$ Henry Kissinger, & À Henry Kissinger, \\
\hline 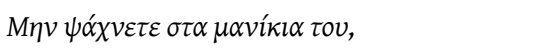 & Ne cherchez pas dans ses manches, \\
\hline 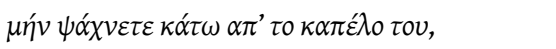 & ne cherchez pas sous son chapeau, \\
\hline 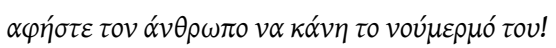 & laissez l'homme faire son numéro! \\
\hline
\end{tabular}

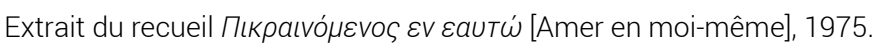

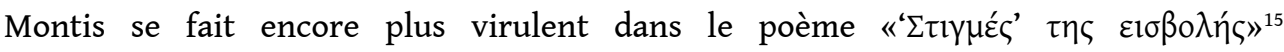
[« Moments » de l'occupation], extrait du même recueil. Évoquant Hitler et l'holocauste il ose l'invective raciale à l'encontre du diplomate américain.

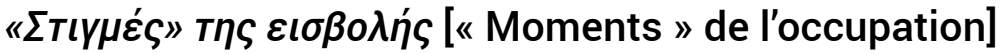

\begin{tabular}{|c|c|}
\hline$\Sigma \tau o$ Henry Kissinger, & À Henry Kissinger, \\
\hline 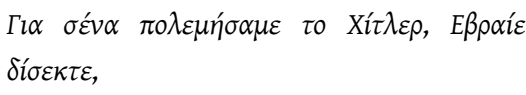 & $\begin{array}{l}\text { Nous avons combattu Hitler pour toi, Juif de mauvais } \\
\text { augure, }\end{array}$ \\
\hline 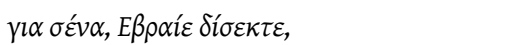 & pour toi, Juif de mauvais augure, \\
\hline
\end{tabular}




\begin{tabular}{|c|c|}
\hline 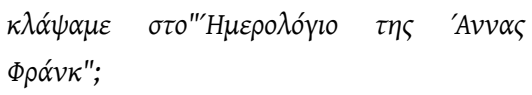 & nous avons pleuré sur le « Journal d'Anne Frank»; \\
\hline 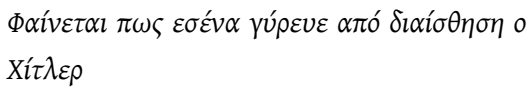 & Il semble qu'Hitler ait eu pour toi de l'intuition \\
\hline 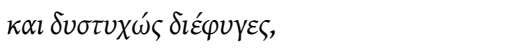 & et malheureusement tu lui as échappé \\
\hline 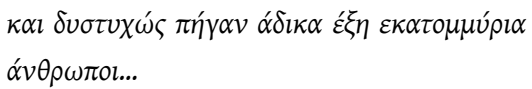 & $\begin{array}{l}\text { et malheureusement six millions de personnes ont péri } \\
\text { injustement... }\end{array}$ \\
\hline
\end{tabular}

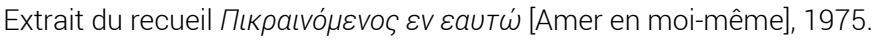

\section{Conclusion}

J'ai voulu par ce rapide panorama montrer comment la poésie de Montis accompagne, avec la sensibilité de l'auteur les grands événements qui ont bouleversé l'histoire récente de Chypre. Elle met parfois crûment en exergue la colère du poète engagé. Elle est enfin un saisissant reflet de préoccupations qui marquent encore aujourd'hui la communauté chypriote grecque.

\section{BIBLIOGRAPHIE}

BOUVIER Bertrand, LAZARIDIS Anastasia Danaé, 1984, «Improvisateurs Chypriotes : une journée avec les poètes populaires des kokkinochoria ", in Actes du VIII ${ }^{\mathrm{e}}$ Congrès International des Néohellénistes des Universités francophones, Montpellier 10-11-12 mai 1984.

DANIELIDOu Liana, HORVATH Peter, 2006, “Greek Cypriot Attitudes Toward Turkish Cypriots and Turkish Immigrants", in The Journal of Social Psychology, no 146, vol. 4, Heldref Publications, pp. 405-421, http://academos.ro/sites/default/files/biblio-docs/207/journalofsocialpsychology.pdf [consulté le 23 mars 2016].

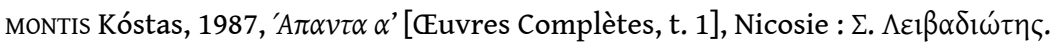

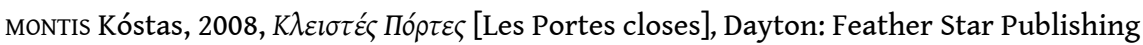
(réédition).

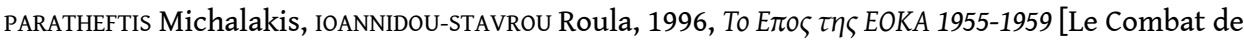

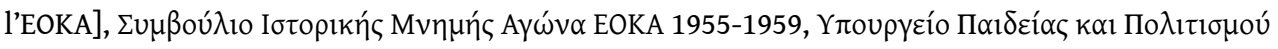
[Conseil pour la mémoire historique de la lutte de l'EOKA, Ministère de la Culture et de l'Éducation]. 


\section{NOTES}

1. Ce texte est né d'une intervention à la journée Écritures balkaniques de l'Inalco (sous la direction de Frosa Pejoska-Bouchereau) le 18 décembre 2009.

2. Sur cette tradition orale, voir l'article de Bertrand BOUviER et Anastasia Danaé LAZARIDIS, «Improvisateurs Chypriotes : une journée avec les poètes populaires des kokkinochoria ", in Actes du VIII $^{\mathrm{e}}$ Congrès international des néohellénistes des universités francophones, Montpellier 10-11-12 mai 1984, p. 22.

3. Kóstas Montis s'explique à ce sujet dans les toutes premières pages des Portes closes :

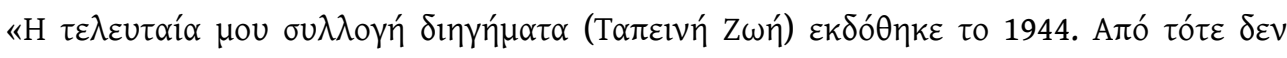

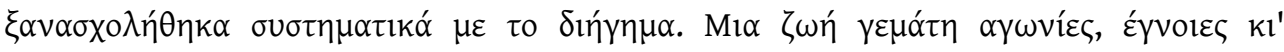

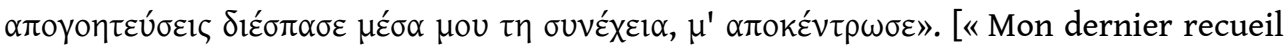
de nouvelles est paru en 1944. Depuis je ne suis plus penché systématiquement sur l'écriture de nouvelles. Une vie de combats, de soucis, et de déceptions a rompu en moi

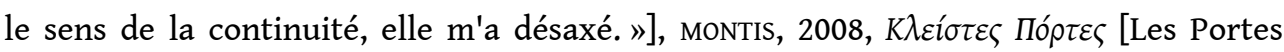
closes], p. 5.

4. Le texte du poème est plus long, il dénonce l'inhumanité des forces britanniques d'occupation. Michalakis KARAOLIS in PARATHEFTIS Michalakis, IOANNIDOU-STAVROU Roula,

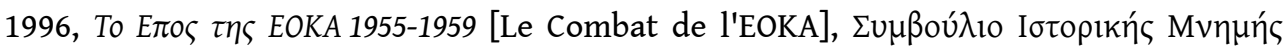

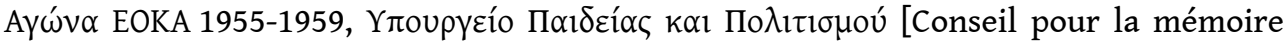
historique de la lutte de l'EOKA, Ministère de la Culture et de l'Éducation], p. 55.

5. MONTIS, p. 66.

6. Lecture par le poète, extraits de 'A $\alpha \alpha v \tau \alpha$ [Æuvres Complètes], film disponible sur www.costasmontis.com

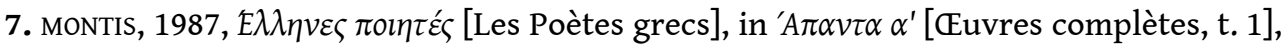
p. 456.

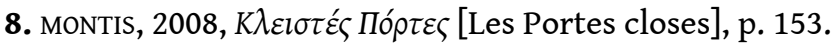

9. MONTIS, p. 149.

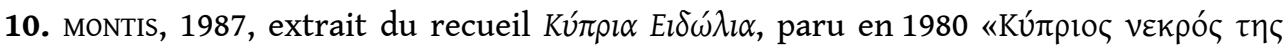

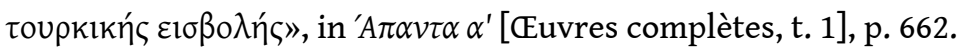

11. Jusqu'en 2003, c'était le seul point de passage entre les deux parties de l'île, et le bâtiment est aujourd'hui le siège de la FNUCYP, présente depuis 1964.

12. DANiELIDOu Liana, HoRvath Peter, 2006, "Greek Cypriot Attitudes Toward Turkish Cypriots and Turkish Immigrants", in The Journal of Social Psychology, no 146, vol 4, Heldref Publications, pp. 405-421.

13. MONTIS, Unrevealed statue [La Statue non dévoilée], p. 159.

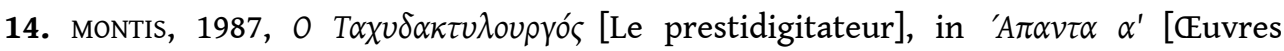
Complètes, t. 1], p. 569.

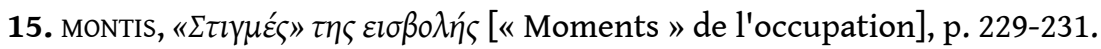




\section{RÉSUMÉS}

Kóstas Montis est un auteur emblématique de la poésie chypriote grecque du $\mathrm{Xx}^{\mathrm{e}}$ siècle, l'un des plus connus et célébrés; sa poésie, comme celle de nombreux de ses confrères chypriotes reflète les passions et tragédies de l'île, la ferveur de la lutte pour l'indépendance, la douleur et la colère, les espoirs de réunification et la mise en avant d'une identité commune entre Chypriotes grecs et turcs.

Costas Montis is a leading writer of the Greek-Cypriot poetry of the 20th century, one of the most well-known and celebrated; his poetry, as the works of others Cypriot poets, reflects the passions and tragedies of the island, the height of the fight for independency, the pain and anger, the hope for reunification and the highlighting of a common identity between Greek and Turks Cypriots.

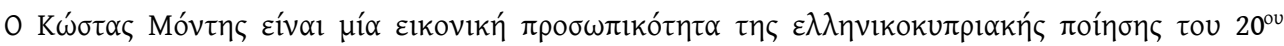

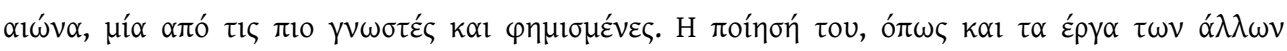

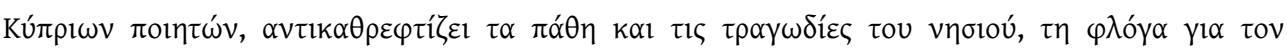

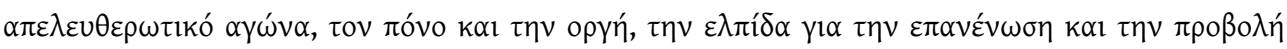

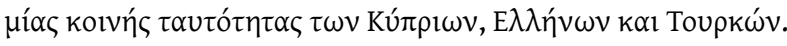

\section{INDEX}

Thèmes : Littérature grecque, Poésie, Histoire et littérature

Mots-clés : Montis Kóstas (1914-2004), Montis Kóstas (1914-2004)

Keywords : Montis Costas (1914-2004), Cyprus, Twentieth Century, Greek literature, Poetry, History and literature

motsclesmk МОНТИС КОСТАС (1914-2004), КИПАР, ДВАЕСЕТТИОТ ВЕК, ГРЧКАТА ЛИТЕРАТУРА, ПОЕЗИЈА, ИСТОРИЈА И ЛИТЕРАТУРА

motsclestr Montis Kostas (1914-2004), Kıbrıs, Yirminci yüzyıl, Yunan Edebiyatı, şiir, Tarih ve Edebiyat

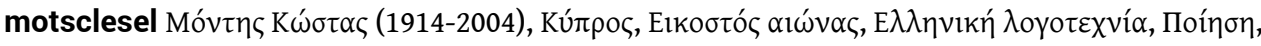

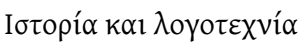

Index géographique : Chypre

Index chronologique : vingtième siècle

\section{AUTEUR}

\section{ALEXANDRE LAPIERRE}

CREE, Inalco, Sorbonne Paris Cité 